\title{
Instructional Design for Student Learning on Quantum Tunneling
}

\author{
Shobha Kanta Lamichhane \\ Department of Science Education, Prithvi Narayan Campus, Pokhara \\ Correspondence: sklamichhane@hotmail.com
}

\begin{abstract}
Acquiring mathematical, conceptual, and problem-solving skill is required in university-level physics courses. Average students often lack the knowledge and study skills need to succeed in physics, preciously in quantum physics course. The reasons for these difficulties are more subtle and some of them are brought to the surface. Addressing how to build models of wave functions and energy and how to relate these models to real physical systems is a challenging job. Article has opened up a floodgate of deep and difficult task for students struggle to make sense of these models. Article has discuss the difficulties and the real issues of student in learning quantum tunneling to build the models that are implicit in experts' understanding. So that, in addition to class attendance and/or group study, students must also learn to work by themselves to develop the mathematical, conceptual, and problem-solving skills they need.
\end{abstract}

Keywords: quantum tunneling, uncertanity, padagogy, process skills.

\section{Introduction}

Quantum tunneling is one of the novel implications of Quantum Mechanics (QM). Tunneling is explain using the Heisenberg uncertainty principle and the wave-particle duality of matter. It is a surprising result that has serve to validate the theory of QM by explaining many real world phenomena such as alpha decay, molecular bonding, and field emission and has resulted in applications such as STM (fig. 5). As a case study in the counter intuitive yet applicable nature of QM, tunneling is an important part of any introductory course in QM. An examination of modern physics and QM textbooks, course syllabi, and interviews with faculty who have taught such courses suggest that instruction in tunneling should help students achieve the following learning goals: (1) calculate or discuss qualitatively, the probability of tunneling for various physical situations, (2) describe the meaning of the potential energy and wave-function graphs, (3) visualize how these graphs would change if the physical situation were altered, e.g., changing barrier height and width, and (4) relate the mathematical formalism and graphical representation of tunneling to the phenomenon of tunneling in the real world.

Tunneling has been a favorite topic of physics education researchers specializing in QM, who have found that many students have a great deal of trouble understanding even the most basic aspects of this topic. [1-7] In designing a transformed course in modern physics for engineering majors, [8] we drew on the literature of previous research to develop a curriculum aimed at addressing known student difficulties in understanding quantum tunneling.[9] Throughout the process of developing and refining this course, we carried out a study to answer the following research questions:

(1) does our curriculum help to address common student difficulties in learning tunneling?

(2) are our students achieving the learning goals describe above?

(3) what are the practices that support or hinder the achievement of these goals?

We find that ourcurriculum does help students overcome common difficulties and achieve our learning goals. While the common difficulties reported in the literature do arise in the transformed classes, they are less prevalent than in comparable traditional classes, and they often arise for different reasons than discussed in 
the previous literature. Further, we find new difficulties that have not been previously reported, associated with a struggle to make sense of the models of QM and relate them to the real world. The difficulties discussed in the literature are associated with the inability to apply the quantum model to abstract model systems such as square barriers and square wells. Our existing course focuses on relating these abstract model systems to reality, and this study shows that the difficulties discussed in the literature are surface features, masking a much more serious problem: In tunneling, as in other aspects of QM, students fail to grasp the basic models that we are using to describe the world as anything more than abstract model systems. These models include wave functions as descriptions of physical objects, potential energy graphs as descriptions of the interactions of those objects with their environments, and total energy as a delocalized property of an entire wave function that is a function of position. Thus, even when students can successfully overcome problems that previous research has elucidated, such as relating wave functions to potentials, they may not know what a wave function or a potential is.

Hestenes [10] pointed out that while "a physicist possesses a battery of abstract models with ramifications already worked out or easily generated," standard physics instruction often treats these models implicitly rather than explicitly. While this is true even in introductory physics, the problem is more serious in $\mathrm{QM}$, where the models are particularly abstract, and the connection between the models and the real world is more tenuous. Standard instruction in QM, including tunneling, does not provide students with enough information to make sense of these models, to relate them to anything real, or even to recognize that they exist. We have achieved a degree of success in teaching.

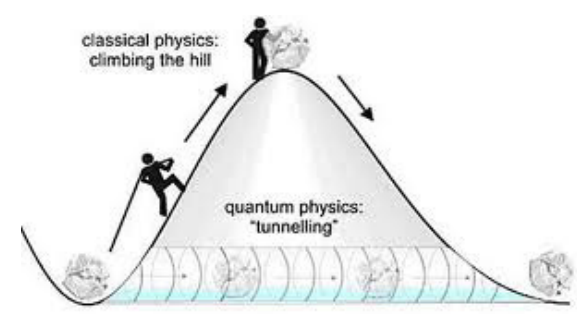

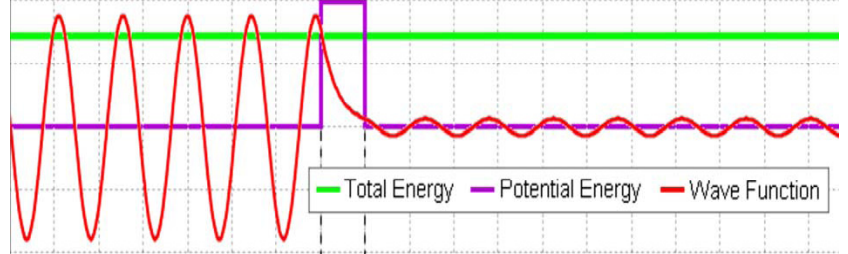

Fig. 1. The standard presentation of quantum tunneling: a plane wave tunneling through a square potential barrier. Total energy, potential energy, and the real part of the wave function are all drawn on the same graph, and the real part of the wave function is labeled as simply wave function.

\section{Methodology}

The qualitative data were collected that consist of observations of students in lectures and problemsolving sessions, student responses to essay questions on homework and exams, and student interviews. Interviews included students participating in a case study project, interviews on the Quantum Mechanics Conceptual Survey (QMCS), [37, 38] which include questions on tunneling, ${ }^{7}$ and interviews on the Quantum Tunneling and Wave Packets simulation. The quantitative data consist of student responses on the QMCS, homework, and exams. Observations included approximately 70 lectures (20 on tunneling) and 50 problem-solving sessions (5 on tunneling). In lectures, researcher took detailed field notes during and after class, writing down all questions that students asked the lecturer and summarizing student discussions during tick questions. The researcher also took field notes immediately after problem-solving sessions, writing summaries of the interactions with and observations of students working on homework. Taken help from junior faculty members to facilitate student discussion during lecture and problem-solving sessions also took field notes, that provided an additional perspective.

\section{Results and Discussion}

\section{Standard representation of quantum tunneling}

Matter, in QM is behaving as properties of waves and particles. One interpretation of this duality involves the Heisenberg uncertainty principle, which defines a limit on how precisely the position and the momentum of a particle can be known at the same time [4]. This implies that there are no solutions with a probability of exactly zero (or one), though a solution may approach 
infinity, for example, the calculation for its position was taken as a probability of 1 , the other, ie its speed would have to be infinity. Hence, the probability of a given particle's existence on the opposite side of an intervening barrier is non-zero, and such particles will appear- with no indication of physically transiting the barrier-on the 'other' side with a frequency proportional to this probability.

Tunneling is defined as a wave function passing through a potential-energy barrier that is greater than its total energy. The typical presentation includes an analysis of the plane wave solution to the Schrödinger equation for a square potential-energy barrier, as shown in Fig. 1. Often the wave function, potential energy, and total energy are drawn on the same graph, a practice which research has shown to lead to student confusion $[3,6]$ and which thoughtful authors have avoided since the 1970s [11]. Depending on the level of the textbook, the reflection and transmission coefficients are either derived or given. This is typically followed by a discussion of some applications of quantum tunneling, such as alpha decay, STM, and the inversion of ammonia molecules. Some textbooks also include a discussion of tunneling wave packets, occasionally showing pictures of a tunneling wave packet taken from a numerical simulation [12]. Wave packets and applications are nearly always relegated to the end of the discussion of tunneling.

In examining the standard presentation of tunneling, one may ask how it aligns with the learning goals. The standard presentation certainly gives students practice in calculating relevant quantities for the case of a plane wave and square barrier, but it does not give students the tools to extend these calculations to more realistic systems. It also includes both a mathematical model and a discussion of physical applications of this model. However, we argue that it does not provide sufficient links between the two. For example, there is almost never a discussion of what physical system could produce the square barrier shown in Fig. 1 or of how a plane wave relates to a real particle. Further, when real applications are discussed, their potentialenergy graphs are often not discussed, making it harder for students to relate the applications to the mathematical model. Thus, the standard presentation does not provide students with the tools to extend the model of tunneling beyond square barriers to the more complicated potentials involved in real physical systems, either quantitatively or qualitatively.

\section{Educational findings about quantum tunneling}

Many researchers have documented student difficulties in learning quantum tunneling [1-7]. These researchers, working at many institutions in the United States and Sweden, have found a fairly consistent list of student difficulties. The most common difficulty, discussed in all these references, is the belief that energy is lost in tunneling. The correct description of energy in quantum tunneling is that because there is no dissipation in the Schrödinger equation, energy is conserved, as can be seen in Fig. 1, where the total energy is constant throughout. The barrier itself represents the potential energy, which is zero on the left and right and some positive constant inside the barrier [13]. The kinetic energy is equal to the total energy on the left and right and is negative inside the barrier. Ambrose [1] and Bao [2] reported the student belief that kinetic energy is lost in tunneling, although later research shows that this difficulty is not limited to kinetic energy: Morgan et al. [3] quoted students as saying that "energy" is lost, without specifying which kind of energy, and in our own work, we found that most students who thought that energy is lost did not have a clear idea of which energy is lost. When asked, they were just as likely to say potential, kinetic, or total energy and often used two or even all three types of energy interchangeably within the same explanation [7].

There are two common explanations in the literature for the belief that energy is lost in tunneling. The first explanation (reason 1), attributable to the fact that most textbooks and lecturers draw the energy and the wave function on the same graph, is that students confuse the two, believing that the energy, like the wave function, decays exponentially during tunneling $[2,3,6]$. This explanation is reminiscent of the classic confusion between velocity and acceleration in introductory physics; [14-19] while students can correctly recite definitions and formulas for wave function and energy, they fail to distinguish between the two when solving 
problems.

The second explanation (reason 2) is that students think that 'work' is done on or by the particles while inside the potential barrier [1] or that energy is "dissipated" as in a physical macroscopic tunnel [3]. Many researchers report on student interviews showing that both these explanations are common among students [1-7]. (a)

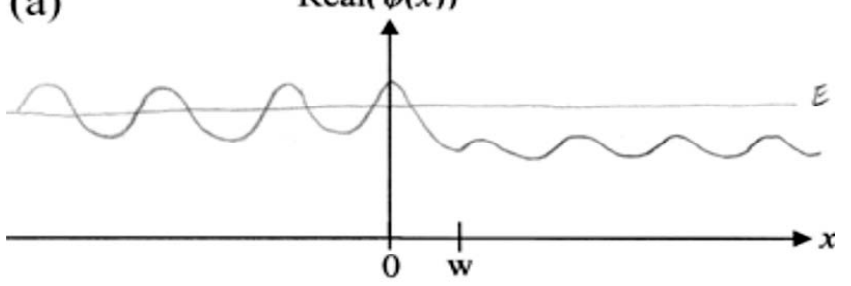

(b)

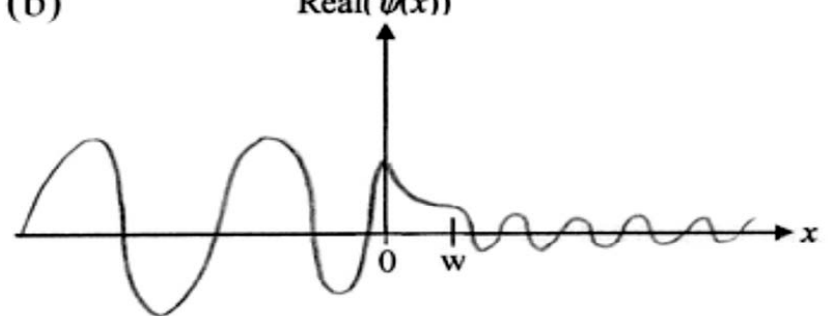

Fig. 2. Drawing the real part of the wave function with (a) an offset between the horizontal axes on the left and right sides of the barrier and (b) a smaller wavelength on the right than on the left. These drawings are taken from student responses to an exam question asking students to draw the real part of the wave function.

A third possible explanation (reason 3) suggested by $\mathrm{Bao}^{2}$ is that students may be thinking of mechanical or electromagnetic waves, in which the energy of the wave is related to the amplitude. However, no evidence is presented to support this explanation of student thinking. In our observations and interviews in traditional modern physics courses, few students have sufficient understanding of mechanical or electromagnetic waves to cause problems in their interpretation of the amplitude of matter waves, and none have used such an explanation. As discussed, we do see some evidence of students using this explanation for energy loss in our transformed modern physics course, in which the dependence of amplitude on energy in electromagnetic waves is heavily stressed.

Other common student difficulties reported in the literature are the belief that reflection at a barrier is due to particles having a range of energies, [1] incorrectly drawing the wave function with an offset between the horizontal axes of the wave function on the left and right sides of the barrier, as in Fig. 2(a), [3] incorrectly drawing the wave function with a smaller wavelength on the right than on the left, as in Fig. 2(b), $[1,3]$ and misinterpreting the meaning of the wavelength and amplitude of the wave function.

In addition to these common student difficulties, in our own previous research we found that many students do not know what the potential-energy graph represents. ${ }^{7}$ Our results from student interviews are supported by many conversations with practicing physicists who report having successfully completed QM courses as students without realizing what a potential well was until much later. We believe that this problem is due to the lack of physical context for potential-energy graphs. We will return to this issue later. Brookes and Etkina $[20,21]$ argued that physicists talk about potential using a metaphor of a physical object, as illustrated by the terms "potential well," "potential barrier," and "potential step." Because these metaphors are implicit and their limitations are not discussed, students have a tendency to overextend them, leading to many of the student difficulties that other researchers have documented. This analysis [20] overlaps with ours in that they also pointed out that physics professors are not explicit in discussing the limitations of models.

\section{Modified teaching on quantum tunneling}

As part of the transformation of a modern physics course for engineering majors,[8] we developed a curriculum for teaching quantum tunneling. The course design was based on physics education research (PER) using interactive engagement techniques such as peer instruction and collaborative homework sessions, focusing on real-world applications, and addressing common student difficulties. The curriculum on quantum tunneling was designed to address common student difficulties. Throughout the course, we emphasized building models and relating them to the real world, asking students in lecture, homework, and exams both to construct their own models and to explain models that had been presented to them. ${ }^{9}$ 


\section{A. Addressing student difficulties with energy loss}

Several aspects of the instruction were designed to address the belief that energy is lost in tunneling. As discussed, two reasons that students believe energy is lost in tunneling: (1) treating energy and wave function interchangeably and (2) invoking dissipation.

To address reason 1, we were careful to draw energy and wave function on separate graphs. However, since the representation in Fig. 1, in which they are plotted on the same graph, is ubiquitous in textbooks and other literature, it is impossible to avoid students being exposed to it. This representation has been so ingrained in us by our own education that we had to be on guard to keep from drawing graphs this way ourselves. Therefore, we also used concept questions (multiple choice questions posed in class that students discuss in small groups and answer using a personal response system) and homework questions to elicit student confusion between energy and wave function and address it directly.

Figure 3 shows an example of a concept question used to address this confusion. To address reason 2 , we emphasized energy conservation and the lack of dissipation in the Schrödinger equation. One key feature of the curriculum was activity based Quantum Tunneling Tutorial developed by Wittmann et al.[22] This tutorial was designed to address the belief that energy is lost in tunneling by asking students to work out the total, kinetic, and potential energies in each region and answer questions about energy conservation.

\section{B. Giving potential energy a physical context}

We also designed our curriculum to address our previous finding that students are often confused by the meaning of the potential-energy function [7]. We consistently gave a physical context for potential-energy functions, presenting square wells and barriers as illustrations of real physical systems, rather than mere abstractions. It is worth noting that it was a great challenge for our team of three expert physicists, including one Nobel Laureate, to think of even a single real physical system represented by a square well or a square barrier. This illustrates that for content that is outside of our area of research, even physicists sometimes do not know how an idealized textbook model can be applied to the real world.

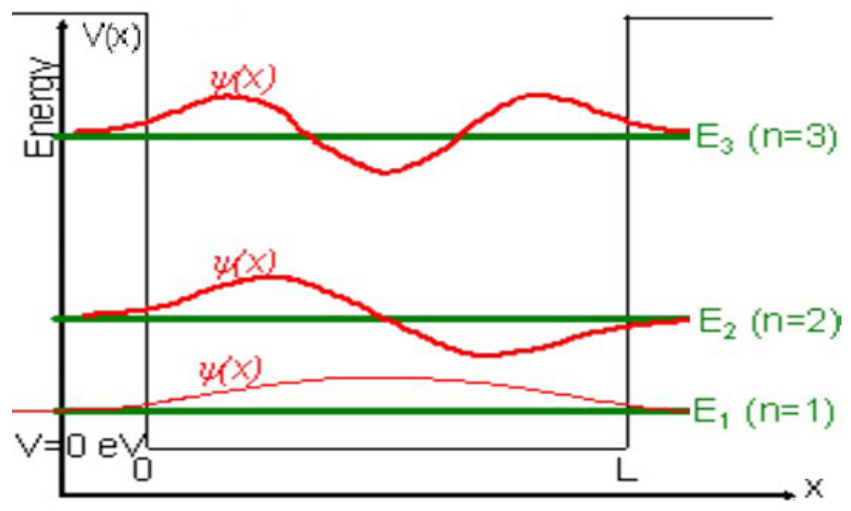

Fig. 3. A concept designed to elicit student confusion between energy and wave function.

The physical examples that we decided to use in our course are illustrated in Fig. 4: an electron in a short wire as the context for a square well and an electron traveling through a long wire with a thin air gap as the context for a square barrier. Because the electrons are free to move around within the wire, the potential energy of an electron is constant anywhere inside the wire (and we can arbitrarily set the constant value to zero). Because the electrons are bound to the wire and require energy to escape, their potential energy outside the wire will be a larger constant, so that the potential energy of the system is well approximated by a finite square well. In lecture, we ask students to predict the value of the potential energy outside the wire by reminding them of the energy required to kick an electron out of a metal in the photoelectric effect, which they learned about earlier in the course.

Students discuss this question in small groups and most eventually recognize that the potential energy outside the wire will be given by the work function of the metal. We chose the physical context of an electron in a wire because it has practical applications for real circuits. While there are a few textbooks that provide physical examples of tunneling, an electron bouncing back and forth between two capacitors with tiny holes in them for a square well [23] and an electron traveling through a series of metal tubes held at different voltages for a square barrier[24], these examples are so artificial that 
no one would ever create such a system for any reason other than to demonstrate the abstract potentials used in introductory QM courses. We decided against using the example of a charged bead moving along a wire held at different potentials that was used in the original version of the Activity-Based Tutorials[22] also because it seemed excessively artificial. Our curriculum included many opportunities for students to practice building models of how potential-energy graphs relate to physical systems. For example, in interactive lectures, homework problems, and a tutorial we asked students to build up potential-energy diagrams for systems such as an electron in a wire, a scanning tunneling microscope, and a nucleus undergoing alpha decay. We also asked students to reason through the physical meaning of the potential energy for various systems. Further, we used the term "potential energy," rather than the shorthand "potential," to avoid confusion[25]. Although it would be preferable to use the symbol $U$, rather than the common convention $V$, for potential energy, to help students relate the potential energy in QM to the potential energy in other areas of physics, we used $V$ in order to be consistent with the textbook we chose for the first semester. However, we repeatedly emphasized the meaning of this symbol and explicitly pointed out the inconsistency in notation among different areas of physics.

(a)
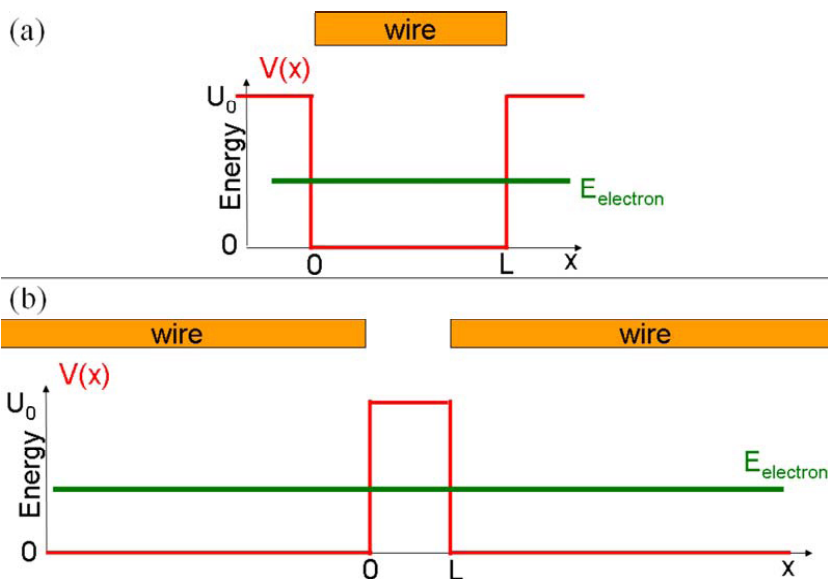

Fig. 4. (a) a square well and (b) a square barrier. A square well with width $L$ and height $U_{0}$ represents a wire with length $L$ and work function $U_{0}$. A square barrier with width $L$ and height $U_{0}$ represents two long wires with work function $U_{0}$ separated by an air gap with length $L$.

\section{Quantum tunneling simulation}

The standard presentation of quantum tunneling provides an abstract and decontextualized model that is difficult to visualize or connect to reality. The content of this presentation is artificially constrained by what can be calculated. Students learn to calculate transmission coefficients for plane waves tunneling through square barriers not because this is a relevant problem but because this is the only tunneling problem that can reasonably be calculated analytically. With modern computational techniques, however, it is no longer necessary for the curriculum to be so constrained. We designed the Quantum Tunneling and Wave Packets simulation[26] (see Fig. 5) to provide easily accessible interactive visual models of tunneling of wave packets and plane waves in a variety of physical situations, thus removing many constraints on curriculum. With the simulation, we can begin our instruction with wave packets, rather than plane waves, so that students can develop a visual model of what is happening in time and space in quantum tunneling. This simulation was developed as part of the Physics Education Technology $(\mathrm{PhET})$ project [27], which provides free interactive computer simulations for teaching physics.
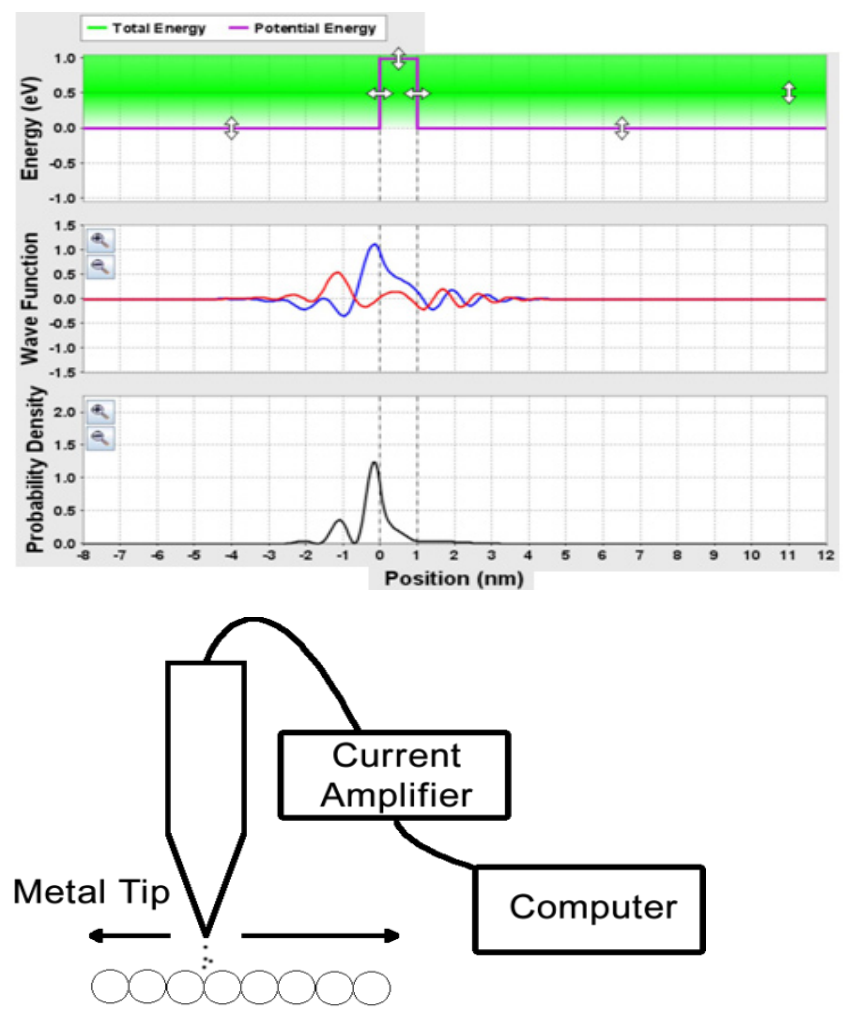

Material Surface 


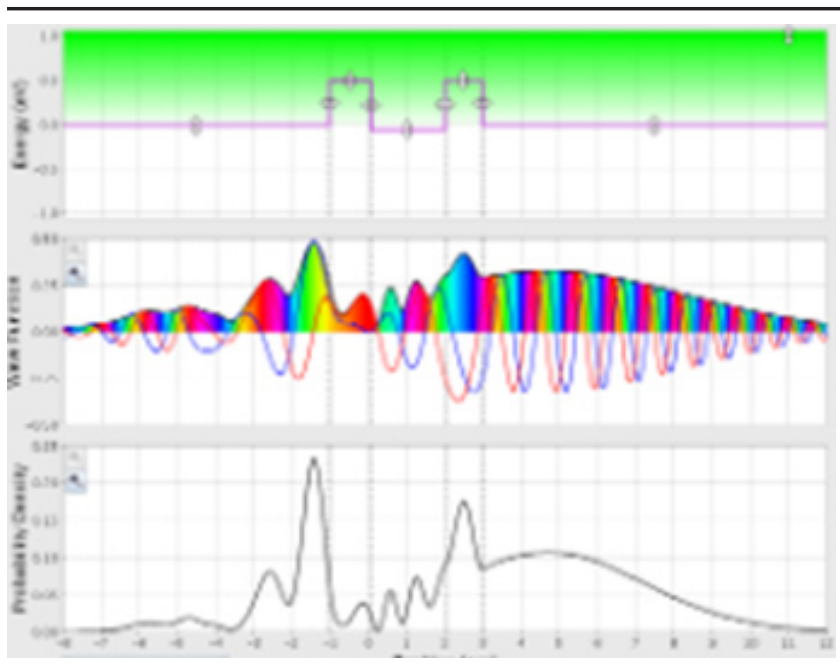

Fig. 5. The Quantum Tunneling and Wave Packets simulation provides interactive visual models of tunneling of wave packets and plane waves in a variety of physical situations

Like other PhET simulations, the Quantum Tunneling and Wave Packets simulation is highly interactive, allowing students to change the potential and total energies by dragging on the graph, so that they can quickly explore a wide variety of physical situations that would be cumbersome to calculate. The simulation also provides a wide variety of representations, allowing students to view the real part, imaginary part, magnitude, and phase of the wave function. To address the problem of students treating energy and wave function interchangeably, these quantities are displayed on separate graphs in the simulation. We note that in the first semester of the reformed course, before developing the simulation, we attempted to use existing simulations on quantum tunneling, as many have already been developed by others [12,28-33]. However, we found that students quickly became frustrated by the limitations of these simulations. For example, students wanted to be able to adjust the properties of the wave packet and/or barrier and to see the real part of the wave function rather than just the magnitude. Further, all these simulations had features that research has demonstrated to be ineffective for student learning, such as plotting the wave function and the potential on the same graph[3,6], using a phase representation[34] and limited interactivity[35].

\section{Conclusion}

Study demonstrates that a focus on addressing common student difficulties is helpful, but not sufficient, for improving student learning of quantum tunneling. By addressing these difficulties and focusing on relating the material to reality, we have uncovered deeper problems in students' ability to use the basic models of QM, such as wave functions as descriptions of physical objects, potential-energy graphs as descriptions of the interactions of those objects with their environments, and total energy as a delocalized property of an entire wave function that is a function of position. We have found that real-world examples are useful not just to help students their lives but also to help them make sense of the models they are using. Effective curriculum on quantum tunneling must explicitly help students learn to build these models. Two practices that we have found useful are focusing on how to relate potential-energy graphs to physical systems and starting with wave packets rather than plane waves. There are several further practices that, although we have not tested them on a large scale. These include (1) tutorials to lead students through the process of drawing potential-energy graphs for various physical situations, (2) explicit discussion of the strengths and weaknesses of gravitational analogies, (3) explicit discussion of the reasons for the focus in QM on an energy representation rather than the force representation used in introductory physics, and (4) explicit discussion of why total energy is quantized (for bound particles) but potential energy is not.

\section{References}

1 R. Knight, 2004. Physics for Scientists and Engineers, (1st ed.) Pearson, San Francisco.

2 C. Cohen-Tannoudji, B. Diu, and F. Laloë, 1977. Quantum Mechanics, Wiley, NY.

3 J. T. Morgan, M. C. Wittmann, and J. R. Thompson, in 2003. Physics Education Research Conference Proceedings. S. Franklin, K. Cummings, and J. Marx, 2004,.AIP, Melville, NY.

4 M. C. Wittmann, J. T. Morgan, and L. Bao, 2005. Addressing student models of energy loss in quantum tunneling, Eur. J. Phys. 26, 939,

5 K. Krane, 1996. Modern Physics, 2nd ed. Wiley, New York.

6 D. Domert, C. Linder, and A. Ingerman, 2005. Probability as a conceptual hurdle to understanding 
one-dimensional quantum scattering and tunneling, Eur. J. Phys. 26, 47.

7 S. B. McKagan and C. E. Wieman, 2005. Physics Education Research Conference Proceedings, Exploring Student Understanding of Energy through the Quantum Mechanics Conceptual Survey, edited by P. Heron, L. McCullough, and J. Marx , AIP, New York,

8 S. B. McKagan, K. K. Perkins, and C. E. Wieman, 2006. Physics Education Research Conference Proceedings, Reforming a large lecture modern physics course for engineering majors using a PER-based design, edited by L. McCullough, P. Heron, and L. Hsu, AIP, New York.

9 Course materials available online at http://per.colorado. edu/ modern

10 D. Hestenes, 1987 . Toward a modeling theory of physics instruction, Am. J. Phys. 55, 440.

11 A. P. French and E. F. Taylor, 1978. An Introduction to Quantum Physics, Norton, New York.

12 A. Goldberg, H. M. Schey, and J. L. Schwartz,1967. Computergenerated motion pictures of onedimensional quantummechanical transmission and reflection phenomena, Am. J. Phys. 35, 177.

13 It is linguistically awkward to speak of the potential energy "inside the barrier" since the potential energy is the barrier, but it is important to be explicit, as many students do not recognize the equivalence of potential energy and barrier.

14 L. Viennot,1979. Spontaneous reasoning in elementary dynamics, Eur. J. Sci. Educ. 1, 205.

15 D. E. Trowbridge and L. C. McDermott, 1980. Investigation of student understanding of the concept of velocity in one dimension, Am. J. Phys. 48, 1020.

16 D. E. Trowbridge and L. C. McDermott, 1981. Investigation of student understanding of the concept of acceleration in one dimension, Am. J. Phys. 49, 242.

17 J. Clement, Students', 1982. preconceptions in introductory mechanics, Am. J. Phys. 50, 66.

18 I. A. Halloun and D. Hestenes, 1985. Common sense concepts about motion, Am. J. Phys. 53, 1056.

19 I. Galili and V. Bar,1992. Motion implies force: Where to expect vestiges of the misconception? Int. J. Sci. Educ. 14, 63.

20 D. T. Brookes and E. Etkina, 2005. Physics Education Research Conference Proceedings, edited by P. Heron, L. McCullough, and J. Marx ,AIP, Melville, NY.

21 D. T. Brookes, Ph.D. thesis, Rutgers University, 2006.

22 M. C. Wittmann, R. N. Steinberg, E. F. Redish, 2005. University of Maryland Physics Education Research Group, Activity-Based Tutorials Modern Physics, Wiley, New York, Vol. 2.

23 R. Harris, 1998. Nonclassical Physics, (1 ${ }^{\text {st }}$ ed $)$ Pearson,
Menlo Park.

24 R. Eisberg and R. Resnick, 1985. Quantum Physics of Atoms, Molecules, Solids, Nuclei, and Particles Wiley, New York.

25 J. R. Taylor, C. D. Zafiratos, and M. A. Dubson, 2004. Modern Physics for Scientists and Engineers, 2nd ed. Pearson Prentice Hall, Upper Saddle River.

26 H. C. Ohanian, , 1995. Modern Physics, 2nd ed. Prentice, Englewood Cliffs, NJ.

27 R. Shankar, 1994. Principles of Quantum Mechanics, Springer, New York.

28 J. R. Hiller, I. D. Johnson, and D. F. Styer, 1995.Quantum Mechanics Simulations: The Consortium for UpperLevel Physics Software, Wiley, New York.

29 B. Thaller, 2000. Visual Quantum Mechanics, Springer, New York.

30 M. Belloni, W. Christian, and A. J. Cox, 2006. Physlet Quantum Physics: An Interactive Introduction, Pearson Prentice Hall, Upper Saddle River, NJ.

31 R. W. Gurney and E. U. Condon, 1929. Quantum mechanics and radioactive disintegration, Phys. Rev. 33, 127.

32 J. B. Pethica and R. Egdell, 2001. Surface science: The insulator uncovered, Nature, London, 414, 27.

33D. J. Griffiths, 2005. Quantum Mechanics, $\left(2^{\text {nd }}\right.$ ed. Pearson) Upper Saddle River.

34 S. B. McKagan, K. K. Perkins, M. Dubson, C. Malley, S. Reid, R. LeMaster, and C. E. Wieman, 2008. Developing and researching PhET simulations for teaching QM, Am. J. Phys. 76, 406.

35 W. K. Adams, S. Reid, R. LeMaster, S. B. McKagan, K. K. Perkins, and C. E. Wieman, 2008. A study of educational simulations (Part I: engagement and learning) J. Interact. Learn. Res. 19, 397. 